\title{
A Hybrid 3DMLUV-FIA Method for Scattering from a 3D Dielectric Object above a 2D Dielectric Rough Surface
}

\author{
Wu Xuezhi ${ }^{1}$ and Wenping $Y u^{2}$ \\ ${ }^{1}$ Naval University of Engineering, Wuhan, Hubei 430033, China \\ ${ }^{2}$ Wuhan University, Wuhan, Hubei 430072, China \\ Correspondence should be addressed to Wu Xuezhi; 13006308755@163.com
}

Received 25 February 2016; Revised 3 July 2016; Accepted 31 July 2016

Academic Editor: Paolo Burghignoli

Copyright @ 2016 W. Xuezhi and W. Yu. This is an open access article distributed under the Creative Commons Attribution License, which permits unrestricted use, distribution, and reproduction in any medium, provided the original work is properly cited.

\begin{abstract}
The electromagnetic scattering from the composite model of a three-dimensional (3D) dielectric object located above a twodimensional (2D) dielectric rough surface is analyzed in this work. Poggio, Miller, Chang, Harrington, Wu, and Tsai (PMCHWT) integral equations are discretized by the method of moments (MoM) into a matrix which is solved by Biconjugate Gradients Stabilized (BICGSTAB) method. Method of 3DMLUV was used for PEC object located above rough surface. Comparing to the case when object and rough surface are both PEC, the memory requirement and computational complexity for dielectric models are increased due to doubled unknown number. Moreover, compared to dielectric object in free space, the coupling between dielectric object and dielectric rough surface will result in complicated numerical simulation. To solve this problem, the updated rank based 3D Multilevel UV (3DMLUV) method is employed to reduce memory consumption and CPU time overhead. The 3DMLUV has been successfully applied in the scattering of PEC targets; however, when the object or rough surface becomes dielectric, the coupling between dielectric object and dielectric rough surface will lead to slow constriction. Therefore, the Fast Iterative Approach (FIA) is applied to further speed up the constricted speed of the matrix required in 3DMLUV. The efficiency, stability, and accuracy of the proposed method are demonstrated in a variety of scattering problems.
\end{abstract}

\section{Introduction}

Electromagnetic (EM) scattering from an object above a rough surface has attracted much interest during recent years because of its extensive applications to remote sensing, target recognition, radar surveillance, and so on [1-3]. As a more general practical problem, when object and rough surface are both dielectric, equivalent electric and magnetic currents will emerge in object and rough surface at the same time, which leads to large storage and much more CPU time. Thus, it is of great value to improving rapid and efficient algorithm. For instance, hybrid FE-BI-KA method and FEM/BIE $[4,5]$ method were applied by Guo et al., SAMM [6] method was used by Morgenthaler and Rappaport, and SDFMM [7] method was used by El-Shenawee et al. when calculating the scattering from dielectric surface-body model; integral equation that can solve the induced current and magnetic induction flow at the same time should be proposed, such as
PMCHW [8-11] and CFIE-JMCFIE [12, 13], and solved with MOM.

In this paper, we present a method of moments (MoM [14]) solution of the PMCHWT and EFIE surface integral equations for scattering by 3D, arbitrarily shaped, homogeneous objects above a $2 \mathrm{D}$ rough surface accelerated by hybrid 3DMLUV-FIA method. 3DMLUV [15] is used to compress the coefficient matrices. Fast Iterative Approach (FIA) is further implemented to speed up the constrictive iteration.

The 3DMLUV method is developed by Deng et al. using EM-interaction-based sampling algorithm. It is an efficient technique to analyze large scale scattering problems and show a computation complexity of $O(N \log N)$. The details of the 3DMLUV can be found in [15]. However, when the object or rough surface becomes dielectric, the coupling between dielectric object and dielectric rough surface will lead to oscillatory and slow constriction. The FIA method is 
an iterative updating method with iterative initial value and it can turn mutual interaction into self-interaction; hence, its implementation simplifies the matrices needed in 3DMLUV. Therefore, the FIA method is a perfect choice to speed up the constriction speed in 3DMLUV. As PEC object located above rough surface is widely researched, 3DMLUV-FIA method is a new great method for dielectric object located above a dielectric rough surface.

The remainder of the paper is organized as follows. In Section 2, we present the implementation of the PMCHWT. The Galerkin method is utilized, where RWG functions are used as both basis and testing functions. The 3DMLUV-FIA is presented. In Section 3, the numerical results are shown. The accuracy of the proposed method is validated first. Then, bistatic radar cross section (RCS) of the object and rough surface below the object are calculated. The influence of the medium permittivity on the scattering characteristic is investigated.

\section{Theory}

2.1. MOM Formulation of PMCHW Equation. As shown in Figure 1, a 3D object (dielectric) is located above a 2D random rough surface (dielectric). The air space, the space under rough surface, and the space object occupied are denoted by Region $0\left(R_{0}\right)$, Region $1\left(R_{1}\right)$, and Region $2\left(R_{2}\right)$, while the surface of the object and the rough surface are indicated as $s_{1}$ and $s_{2}$. The three regions have permittivity and permeability given by $\varepsilon_{0}$ and $\mu_{0}, \varepsilon_{1}$ and $\mu_{1}, \varepsilon_{2}$ and $\mu_{2}$, respectively. The electric and magnetic fields in Region 0 , Region 1 , and Region 2 are $E_{0}, H_{0}, E_{1}, H_{1}$, and $E_{2}, H_{2}$.

Using the equivalence theorem, the equivalent electric and magnetic current on rough surface and the surface of objects are $\mathbf{J}_{s}, \mathbf{M}_{s}, \mathbf{J}_{o}, \mathbf{M}_{o}$, respectively.

So, the PMCHW equation can be given by [16]

$$
\begin{aligned}
& \eta_{0} \mathbf{L}_{0}\left(\mathbf{J}_{s}\right)+\eta_{1} \mathbf{L}_{1}\left(\mathbf{J}_{s}\right)+\eta_{0} \mathbf{L}_{0}\left(\mathbf{J}_{o}\right)-\mathbf{K}_{0}\left(\mathbf{M}_{s}\right)-\mathbf{K}_{1}\left(\mathbf{M}_{s}\right) \\
& \quad-\left.\mathbf{K}_{0}\left(\mathbf{M}_{o}\right)\right|_{s_{1}, \tan }=-\left.\mathbf{E}^{\mathrm{inc}}\right|_{s_{1}, \tan } \\
& \mathbf{K}_{0}\left(\mathbf{J}_{s}\right)+\mathbf{K}_{1}\left(\mathbf{J}_{s}\right)+\mathbf{K}_{0}\left(\mathbf{J}_{o}\right)+\frac{1}{\eta_{0}} \mathbf{L}_{0}\left(\mathbf{M}_{s}\right)+\frac{1}{\eta_{1}} \mathbf{L}_{1}\left(\mathbf{M}_{s}\right) \\
& \quad+\left.\frac{1}{\eta_{0}} \mathbf{L}_{0}\left(\mathbf{M}_{o}\right)\right|_{s_{1}, \tan }=-\left.\mathbf{H}^{\mathrm{inc}}\right|_{s_{1}, \tan } \\
& \eta_{0} \mathbf{L}_{0}\left(\mathbf{J}_{s}\right)+\eta_{0} \mathbf{L}_{0}\left(\mathbf{J}_{o}\right)+\eta_{2} \mathbf{L}_{2}\left(\mathbf{J}_{o}\right)-\mathbf{K}_{0}\left(\mathbf{M}_{s}\right) \\
& \quad-\mathbf{K}_{0}\left(\mathbf{M}_{o}\right)-\left.\mathbf{K}_{2}\left(\mathbf{M}_{o}\right)\right|_{s_{2}, \tan }=-\left.\mathbf{E}^{\mathrm{inc}}\right|_{s_{2}, \tan } \\
& \quad \mathbf{K}_{0}\left(\mathbf{J}_{s}\right)+\mathbf{K}_{0}\left(\mathbf{J}_{o}\right)+\mathbf{K}_{2}\left(\mathbf{J}_{o}\right)+\frac{1}{\eta_{0}} \mathbf{L}_{0}\left(\mathbf{M}_{s}\right)+\frac{1}{\eta_{0}} \mathbf{L}_{0}\left(\mathbf{M}_{o}\right) \\
& \quad+\left.\frac{1}{\eta_{2}} \mathbf{L}_{2}\left(\mathbf{M}_{o}\right)\right|_{s_{2}, \tan }=-\left.\mathbf{H}^{\mathrm{inc}}\right|_{s_{2}, \tan } \cdot
\end{aligned}
$$

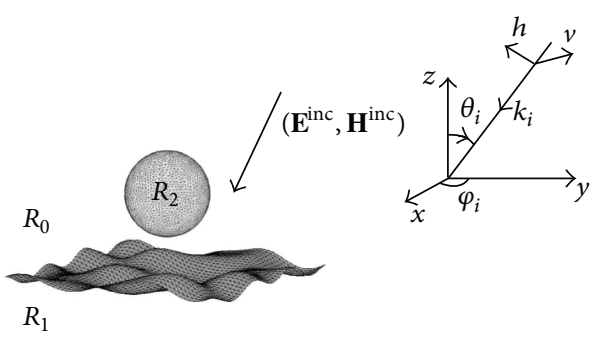

FIGURE 1: Composite scattering model of target and rough surface.

The subscript " $s_{1}$, tan" and " $s_{2}$, tan" are tangential components of the electric field and magnetic field which exist in the surface of rough surface and object.

The electric and magnetic fields at an arbitrary point $r(r$ is the arbitrary distance) in Region 0 are

$$
\begin{aligned}
\mathbf{E}_{0}^{s} & =\eta_{0} \mathbf{L}_{0}\left(\mathbf{J}_{s}\right)-\mathbf{K}_{0}\left(\mathbf{M}_{s}\right)+\eta_{0} \mathbf{L}_{0}\left(\mathbf{J}_{o}\right)-\mathbf{K}_{0}\left(\mathbf{M}_{o}\right) \\
\mathbf{H}_{0}^{s} & =\mathbf{K}_{0}\left(\mathbf{J}_{s}\right)+\frac{1}{\eta_{0}} \mathbf{L}_{0}\left(\mathbf{M}_{s}\right)+\mathbf{K}_{0}\left(\mathbf{J}_{o}\right)+\frac{1}{\eta_{0}} \mathbf{L}_{0}\left(\mathbf{M}_{o}\right),
\end{aligned}
$$

where operators $\mathbf{L}_{i}$ and $\mathbf{K}_{i}$ are given by

$$
\begin{aligned}
& \mathbf{L}_{i}(\mathbf{X})=-j k_{i} \int_{s}\left[\mathbf{X}+\frac{1}{k_{i}} \nabla\left(\nabla^{\prime} \cdot \mathbf{X}\right)\right] G_{i} d s^{\prime} \\
& \mathbf{K}_{i}(\mathbf{X})=-\int_{s} \mathbf{X} \times \nabla G_{i} d s^{\prime}
\end{aligned}
$$

$$
(i=1,2)
$$

The vector $\mathbf{X}$ represents the surface electric current $\mathbf{J}$ and/or the surface magnetic current $\mathbf{M}$ on surface $s_{1}$ or on surface $s_{2} . G_{i}$ is the Green function in homogeneous isotropic medium. $k_{i}=\omega \sqrt{\mu_{i} \varepsilon_{i}}$ is the wave number in Region $i . \eta_{i}=$ $\sqrt{\mu_{i} / \varepsilon_{i}}$.

The equivalent electric and magnetic currents $\left(\mathbf{J}_{s}, \mathbf{M}_{s}\right)$, $\left(\mathbf{J}_{o}, \mathbf{M}_{o}\right)$ are approximated by using the RWG vector basis function $f(r)$ as follows:

$$
\begin{gathered}
\mathbf{J}_{s}=\sum_{n=1}^{N_{s}}\left(I_{1}\right)\left(f_{1}\right)_{n} \\
\mathbf{M}_{s}=\sum_{n=1}^{N_{s}}\left(I_{2}\right)_{n}\left(f_{1}\right)_{n} \\
\mathbf{J}_{o}=\sum_{n=1}^{N_{o}}\left(I_{3}\right)_{n}\left(f_{2}\right)_{n} \\
\mathbf{M}_{o}=\sum_{n=1}^{N_{o}}\left(I_{4}\right)_{n}\left(f_{2}\right)_{n} .
\end{gathered}
$$


$N_{s}$ and $N_{o}$ are the number of unknown coefficients. Upon applying Galerkin method, the original integral equations are thus transformed into a set of linear equations given by

$$
\left[\begin{array}{cccc}
\overline{\bar{Z}}_{s s}^{E J} & \overline{\bar{Z}}_{s s}^{E M} & \overline{\bar{Z}}_{o s}^{E J} & \overline{\bar{Z}}_{o s}^{E M} \\
\overline{\bar{Z}}_{\text {ss }}^{H J} & \overline{\bar{Z}}_{s s}^{H M} & \overline{\bar{Z}}_{o s}^{H J} & \overline{\bar{Z}}_{o s}^{H M} \\
\overline{\bar{Z}}_{s o}^{E J} & \overline{\bar{Z}}_{s o}^{E M} & \overline{\bar{Z}}_{o o}^{E J} & \overline{\bar{Z}}_{o o}^{E M} \\
\overline{\bar{Z}}_{\text {so }}^{H J} & \overline{\bar{Z}}_{s o}^{H M} & \overline{\bar{Z}}_{o o}^{H J} & \overline{\bar{Z}}_{o o}^{H M}
\end{array}\right]\left[\begin{array}{l}
\bar{I}_{1} \\
\bar{I}_{2} \\
\bar{I}_{3} \\
\bar{I}_{4}
\end{array}\right]=\left[\begin{array}{c}
\bar{V}_{s}^{E} \\
\bar{V}_{s}^{H} \\
\bar{V}_{o}^{E} \\
\bar{V}_{o}^{E}
\end{array}\right] .
$$

The dimension of matrix $\overline{\bar{Z}}$ is $2\left(N_{s}+N_{o}\right) \times 2\left(N_{s}+\right.$ $N_{o}$ ). In formula (5), $\bar{I}_{1}$ and $\bar{I}_{2}$ are coefficient of the electricity and magnetic current, respectively, in rough surface, $\bar{I}_{3}$ and $\bar{I}_{4}$ are coefficient of the electricity and magnetic current, respectively, in the surface of object, subscript "ss, so, os, oo" represent the area that source and field exist in when one basis function of RWG plays on others. For instance, "ss" illustrates that the source and field are both at rough surface $s_{1}$, "so" illustrates that source is at rough surface $s_{1}$, while field is at object surface $s_{2}$. Superscript "EJ, EM, HJ, $H M$ " represent the electromagnetic current interaction. $E$ and $H$ indicate electric filed and magnetic field, respectively. $J$ and $M$ indicate electricity and magnetic current in the source-side. Taken together, considering interaction between electricity and magnetic current in rough surface and object, 16 block matrices can be formed. They are as follows: with dimension $N_{s} \times N_{s}$, self-interaction matrices in rough surface- $-\overline{\bar{Z}}_{s s}^{E J}, \overline{\bar{Z}}_{s s}^{E M}, \overline{\bar{Z}}_{s s}^{H J}, \overline{\bar{Z}}_{s s}^{H M}$, with dimension $N_{o} \times N_{o}$, self-interaction matrices in object$\overline{\bar{Z}}_{o o}^{E J}, \overline{\bar{Z}}_{o o}^{E M}, \overline{\bar{Z}}_{o o}^{H J}, \overline{\bar{Z}}_{o o}^{H M}$, with dimensions $N_{s} \times N_{o}$ and $N_{o} \times N_{s}$, interaction between rough surface and object$\overline{\bar{Z}}_{s o}^{E J}, \overline{\bar{Z}}_{s o}^{E M}, \overline{\bar{Z}}_{s o}^{H J}, \overline{\bar{Z}}_{s o}^{H M}, \overline{\bar{Z}}_{o s}^{E J}, \overline{\bar{Z}}_{o s}^{E M}, \overline{\bar{Z}}_{o s}^{H J}, \overline{\bar{Z}}_{o s}^{H M}$.

2.2. Method of 3DMLUV-FIA. At first, we analyze matrix (5) to find which matrix needs compressing. Due to the symmetrical relationship, only three of $\overline{\bar{Z}}_{o o}^{E J}, \overline{\bar{Z}}_{o o}^{E M}, \overline{\bar{Z}}_{o o}^{H J}$, and $\overline{\bar{Z}}_{o o}^{H M}$ will be calculated explicitly [15]. and so it is with $\overline{\bar{Z}}_{s s}^{E J}, \overline{\bar{Z}}_{s s}^{E M}, \overline{\bar{Z}}_{s s}^{H J}$, and $\overline{\bar{Z}}_{s s}^{H M}$. Then, we analyze the rest of eight matrices. They can be expressed as follows:

$$
\begin{aligned}
Z_{s o}^{E J}(m, n) & =\left\langle\left(\vec{f}_{2}\right)_{m}, \eta_{0} \mathbf{L}_{0}\left(\left(\vec{f}_{1}\right)_{n}\right)\right\rangle_{s_{1}} \\
Z_{s o}^{H J}(m, n) & =\left\langle\left(\vec{f}_{2}\right)_{m}, \mathbf{K}_{0}\left(\left(\vec{f}_{1}\right)_{n}\right)\right\rangle_{s_{1}} \\
Z_{s o}^{E M}(m, n) & =-\left\langle\left(\vec{f}_{2}\right)_{m}, \mathbf{K}_{0}\left(\left(\vec{f}_{1}\right)_{n}\right)\right\rangle_{s_{1}} \\
Z_{s o}^{H M}(m, n) & =\left\langle\left(\vec{f}_{2}\right)_{m}, \frac{1}{\eta_{0}} \mathbf{L}_{0}\left(\left(\vec{f}_{1}\right)_{n}\right)\right\rangle_{s_{1}}
\end{aligned}
$$

$$
\begin{aligned}
Z_{o s}^{E J}(m, n) & =\left\langle\left(\vec{f}_{1}\right)_{m}, \eta_{0} \mathbf{L}_{0}\left(\left(\vec{f}_{2}\right)_{n}\right)\right\rangle_{s_{2}} \\
Z_{o s}^{H J}(m, n) & =\left\langle\left(\vec{f}_{1}\right)_{m}, \mathbf{K}_{0}\left(\left(\vec{f}_{2}\right)_{n}\right)\right\rangle_{s_{2}} \\
Z_{o s}^{E M}(m, n) & =-\left\langle\left(\vec{f}_{1}\right)_{m}, \mathbf{K}_{0}\left(\left(\vec{f}_{2}\right)_{n}\right)\right\rangle_{s_{2}} \\
Z_{o s}^{H M}(m, n) & =\left\langle\left(\vec{f}_{1}\right)_{m}, \frac{1}{\eta_{0}} \mathbf{L}_{0}\left(\left(\vec{f}_{2}\right)_{n}\right)\right\rangle_{s_{2}} .
\end{aligned}
$$

Comparing these eight formulas, we can know the following:

$$
\begin{gathered}
\overline{\bar{Z}}_{s o}^{E J}=\eta_{0}^{2} \overline{\bar{Z}}_{s o}^{H M}, \overline{\bar{Z}}_{o s}^{E J}=\eta_{0}^{2} \overline{\bar{Z}}_{o s}^{H M}, \overline{\bar{Z}}_{s o}^{E M}=-\overline{\bar{Z}}_{s o}^{H J}, \overline{\bar{Z}}_{o s}^{E J}= \\
\eta_{0}^{2} \overline{\bar{Z}}_{o s}^{H M}, \overline{\bar{Z}}_{o s}^{E M}=-\overline{\bar{Z}}_{o s}^{H J}, \overline{\bar{Z}}_{o s}^{E J}=\left(\overline{\bar{Z}}_{s o}^{E J}\right)^{T}, \overline{\bar{Z}}_{o s}^{E M}=-\left(\overline{\bar{Z}}_{s o}^{M J}\right)^{T} .
\end{gathered}
$$
So, only two of them are independent and only $\overline{\bar{Z}}_{\text {so }}^{E M}$ and $\overline{\bar{Z}}_{\text {so }}^{E J}$ should be compressed. Above all, upon using 3DMLUV method, only eight submatrices need to be compressed. Among the eight matrices, 2D multidivision is implemented to three independent matrices of rough surface. Then, for submatrices in far-filed, UV decomposition is employed, so it is with three independent matrices of object. Multidivision introduced by Deng et al. [15] is applied to the remaining two matrices of composite scattering.

To improve the constricted speed of matrix (5) when iterating, Fast Iterative Approach is employed. The Fast Iterative Approach of formula (5) can be given by

$$
\begin{aligned}
& \overline{\bar{Z}}_{s s}^{E J} \bar{I}_{1}^{(n)}=V_{s}^{E}-\overline{\bar{Z}}_{s s}^{E M} \bar{I}_{2}^{(n-1)}-\overline{\bar{Z}}_{o s}^{E J} \bar{I}_{3}^{(n-1)}-\overline{\bar{Z}}_{o s}^{E M} \bar{I}_{4}^{(n-1)} \\
& \overline{\bar{Z}}_{s s}^{H M} \bar{I}_{2}^{(n)}=V_{s}^{H}-\overline{\bar{Z}}_{s s}^{H J} \bar{I}_{1}^{(n)}-\overline{\bar{Z}}_{o s}^{H J} \bar{I}_{3}^{(n-1)}-\overline{\bar{Z}}_{o s}^{H M} \bar{I}_{4}^{(n-1)} \\
& \overline{\bar{Z}}_{o o}^{E J} \bar{I}_{3}^{(n)}=\bar{V}_{o}^{E}-\overline{\bar{Z}}_{s o}^{E J} \bar{I}_{1}^{(n)}-\overline{\bar{Z}}_{s o}^{E M} \bar{I}_{2}^{(n)}-\overline{\bar{Z}}_{o o}^{E M} \bar{I}_{4}^{(n-1)} \\
& \overline{\bar{Z}}_{o o}^{H M} \bar{I}_{4}^{(n)}=\bar{V}_{o}^{H}-\overline{\bar{Z}}_{s o}^{H J} \bar{I}_{1}^{(n)}-\overline{\bar{Z}}_{s o}^{H M} \bar{I}_{2}^{(n)}-\overline{\bar{Z}}_{o o}^{H J} \bar{I}_{3}^{(n)},
\end{aligned}
$$

where $n$ is the iterative steps. In the paper, $n$ is the iterative steps when the iterated $I_{1}, I_{2}, I_{3}$, and $I_{4}$ become converged.

During the iteration, firstly update the excitation at the right-hand side of (7a), (7b), (7c), and (7d) with $I_{2}^{n-1}, I_{3}^{n-1}$, and $I_{4}^{n-1}$ obtained in the previous step. Then solve (7a), (7b), (7c), and (7d), separately, to obtain the new currents $I_{1}, I_{2}$, $I_{3}$, and $I_{4}$. The iteration begins with $I_{2}^{(0)}=I_{3}^{(0)}=I_{4}^{(0)}=0$ and is terminated when the iterated $I_{1}, I_{2}, I_{3}$, and $I_{4}$ become converged.

In FIA method, the iterative deviation of the $n$th iteration is defined as $\varsigma_{n}$ :

$$
\varsigma_{n}=\frac{\left\|\overline{\bar{Z}} \bar{I}^{(n)}-\bar{V}\right\|_{2}}{\|\bar{V}\|_{2}}
$$


where

$$
\bar{I}^{(n)}=\left[\begin{array}{l}
\bar{I}_{1}^{(n)} \\
\bar{I}_{2}^{(n)} \\
\bar{I}_{3}^{(n)} \\
\bar{I}_{4}^{(n)}
\end{array}\right] ;
$$

when $\varsigma_{n}=8 e^{-4}$, we think it becomes converged.

The Fast Iterative Approach has two advantages. On one hand, the Fast Iterative Approach deals with interaction between the complex target and the rough surface by method of iteration, and the iteration is divided into two parts, inner loop and outer loop. In the outer loop, the previous iterative value updates incentive vector on the right. In the inner loop, the separate rough surface matrix equation and the single target matrix equation are, respectively, solved, and the initial iterative value of inner loop is set to the previous iterative value of outer loop. In this way, when the outer loop converges very quickly, the calculation of mutually corresponding matrix-vector product between target and rough surface can be greatly reduced. On the other hand, in each iteration step of outer loop, EM scattering between rough surface and target can be decomposed into single target and single rough surface question, which, respectively, corresponds to two matrix equations with smaller dimensions, so that the equations can be quickly solved. There the two loops are just right-hand side of (7a), (7b), (7c), and (7d) and left-hand side of (7a), (7b), (7c), and (7d). we divided the iteration into two loops for showing how and why the FIA method converges quickly.

For the four equations, incentive items which needed updating in the right of the equality are the interaction between electricity and magnetic current and interaction between rough surface and object. It is known form [3] that the interaction of those items is small so that few external iterative loops are needed to meet constriction aim. For inner loops, electric field integral equation (EFIE) like (7a) and (7c) will be solved by Biconjugate Gradients Stabilized (BICGSTAB) method which uses disgonalizable matrices as preprocessed conditions. With high constricted speed, magnetic field integral equation (MFIE) like (7b) and (7d) will be solved by Biconjugate Gradients Stabilized (BICGSTAB) method directly. Comparing to self-iterative matrices, the size of mutual iterative matrices is beyond smaller, which leads to fast constriction of outer loops. The method turns matrices of $2 N \times 2 N$ into matrices of $N \times N$, which significantly decreases the outer loops. The four equations show the simplified process-turn mutual interaction into self-interaction.

\section{Results and Discussions}

In this section, by investigating individual dielectric 3D object, individual dielectric rough surface, and composite model of a 3D object located above 2D Gaussian dielectric rough surface, we can test the accuracy and efficiency of 3DMLUV-FIA method. The CPU employed below is

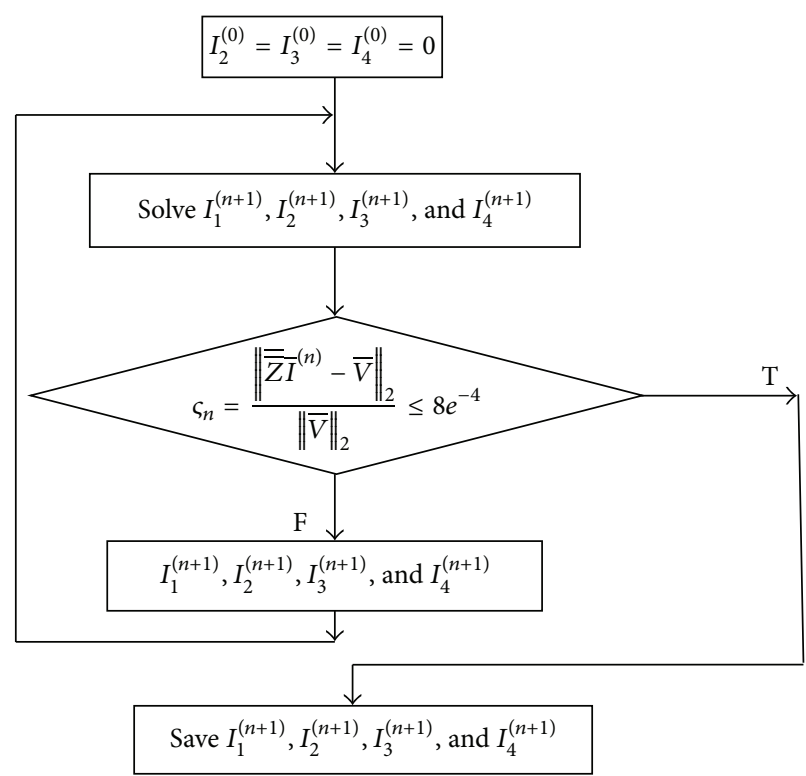

FIgURE 2: The architecture of FIA.

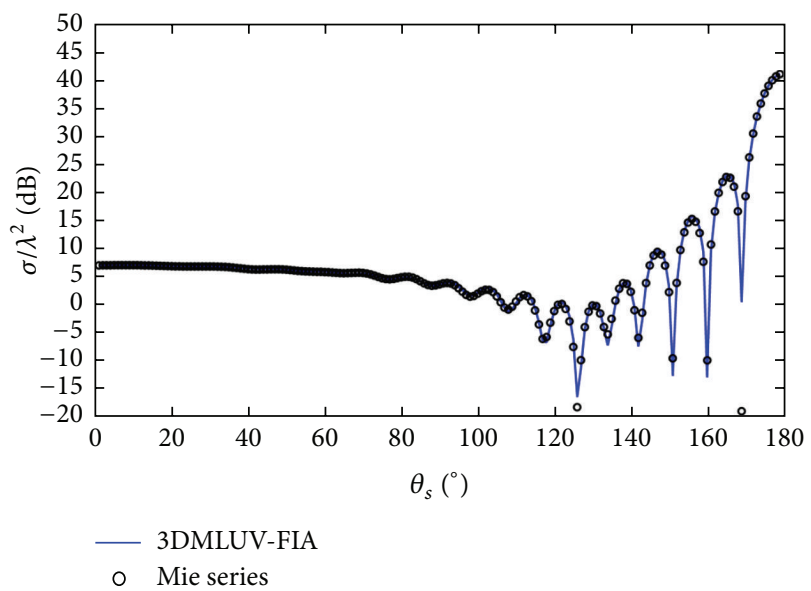

FIGURE 3: Permittivity $\varepsilon_{r}=4-3 j, r=3 \lambda$, RCS from dielectric sphere.

$1.83 \mathrm{GHz}$, processor with $2 \mathrm{G}$ Bytes of RAM. Computational procedure is Intel Fortran compiler 11.

\subsection{Scattering from 3D Dielectric Object}

Case 1. PMCHW equation and 3DMLUV-FIA are implemented in this example. To validate the accuracy of 3DMLUV-FIA solution of PMCHWT integral equations, for plane incident wave, the bistatic RCS of an average dielectric sphere with radius of $r=3 \lambda$ ( $\lambda$ is the wavelength in free space) in free space is calculated and compared with Mie series in Figure 2. The relative permittivity $\varepsilon_{r}=4-3 j$. The numerical result of iterative steps and iterative deviation is displayed in Figure 3.

$n$ in Figure 4 represents the iterative steps. Comparing MFIE (such as formulas (7b) and (7d)) with EFIE (such as formulas (7a) and (7c)), the iterative speed of MFIE is much 

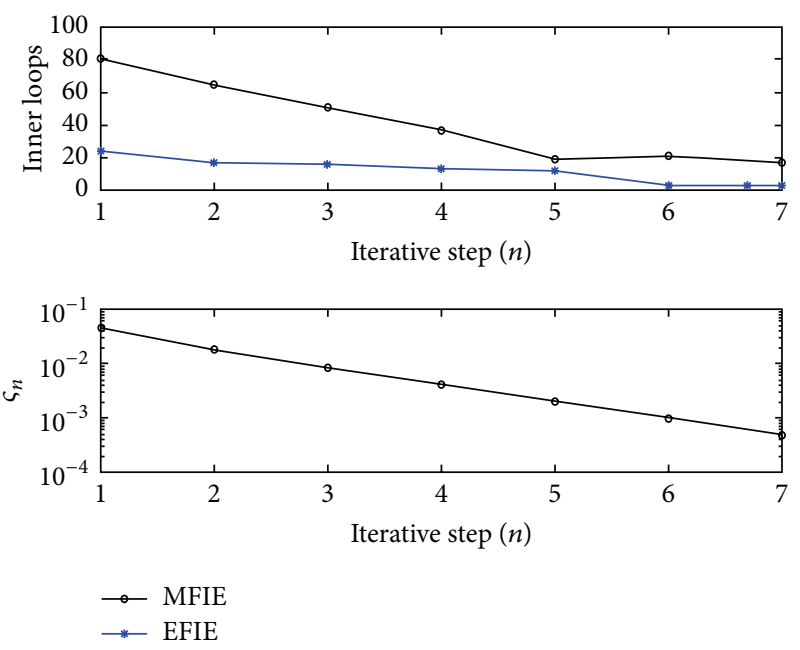

FIGURE 4: Inner loops and iterative deviation $\varsigma_{n}$ vary from iterative steps $n$.

faster than EFIE. We can know from Figure 3 that 3DMLUVFIA has merely the same effect as Mie. For efficiency analysis, the 3DMLUV-FIA is also used to calculate the scattering of the same sphere. From Figure 4, it can be concluded that mutual iteration shows a fast speed when solving PMCHW matrix equation. Moreover, its inner loops reduce with the increasing iterative steps.

3.2. Scattering from Rough Surface. Using the Monte Carlo method, a Gaussian randomly rough surface with given correlation length is generated. The relationship between the truncated surface length $L$ and the surface correlation length $l$ in a $2 \mathrm{D}$ model should satisfy $L \geq 15 l$ [17], and 3D Gaussian randomly rough surface can be generated from a given spectral density and a correlation function [18]:

$$
\begin{gathered}
W\left(K_{x}, K_{y}\right)=\frac{h^{2} l_{x} l_{y}}{4 \pi} \exp \left(-\frac{K_{x}^{2} l_{x}^{2}+K_{y}^{2} l_{y}^{2}}{4}\right) \\
C(x, y)=h^{2} \exp \left(-\frac{x^{2}}{l_{x}^{2}}-\frac{y^{2}}{l_{y}^{2}}\right),
\end{gathered}
$$

where $K_{x}=2 \pi x / L_{x}, K_{y}=2 \pi y / L_{y}, h$ is the root mean square of random surface height, and $l_{x}$ and $l_{y}$ are the correlation lengths along the $x$ and $y$ direction.

The advantage of choosing Gaussian random distribution rough surface is that by adjusting correlation length and root mean square of random surface height, the roughness of random rough surface can be precisely controlled.

In order to eliminate the edge effect caused by the limited truncation of the rough surface, the taped wave is selected instead of a plane wave [19].

Case 2. Consider a 2D dielectric rough surface.

We select Gaussian random distribution rough surface, $\varepsilon_{r 1}=2.5-i 0.18$; the humidity of soil (Bosnian soil) is $3.8 \%$; the incident wave frequency is $f=1 \mathrm{GHz}$.

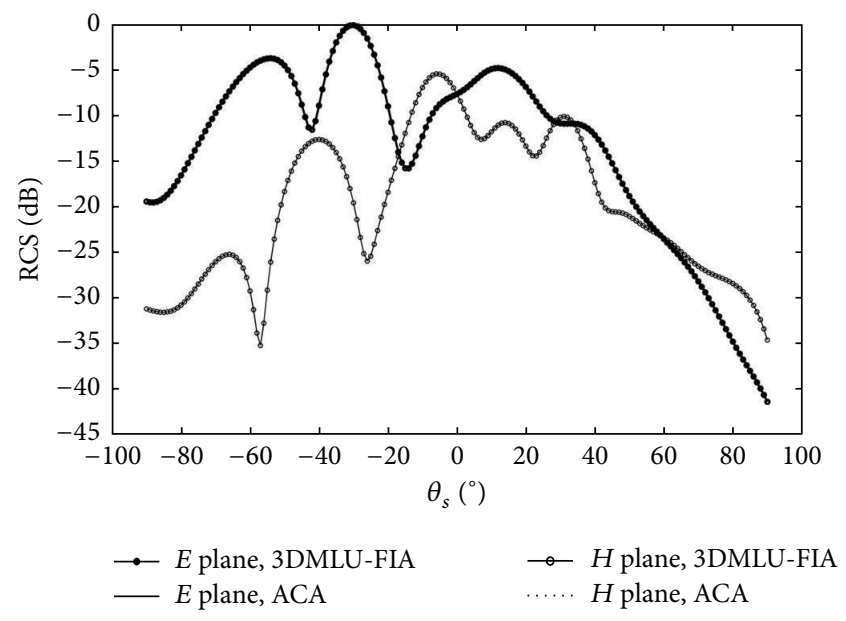

FIGURE 5: The parameters of rough surface: $h=0.1 \lambda, \varepsilon_{r 1}=2.5-$ i0.18, DRCS from dielectric rough surface based on 3DMLUV-FIA method and ACA method.

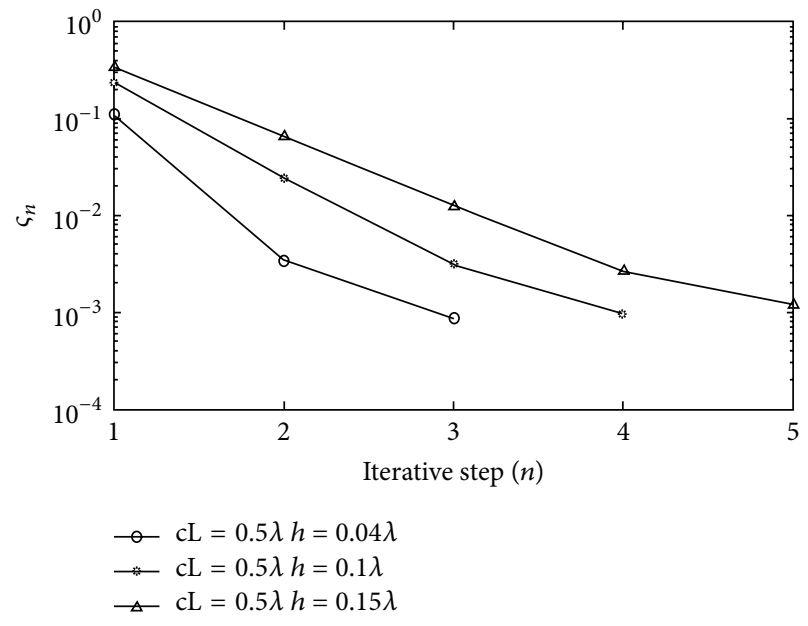

Figure 6: With different RMS height $h=\left[\begin{array}{lll}0.04 & 0.1 & 0.15\end{array}\right] \lambda$, iterative deviation $\varsigma_{n}$ versus iterative steps $n$.

The tapered incident wave with incidence angle $\theta_{i}=30^{\circ}$ is used for all experiments below, given surface length $L_{x}=$ $L_{y}=8 \lambda$, correlation length $l_{x}=l_{y}=\mathrm{cL}=0.5 \lambda$, RMS height $h=\left[\begin{array}{lll}0.04 & 0.1 & 0.15\end{array}\right] \lambda$, and the relative permittivity $\varepsilon_{r 1}=2.5-$ i0.18.

Figure 5 presents, when $h=0.1 \lambda$, the $H H$-polarized bistatic RCS from dielectric rough surface. We first validate the accuracy of 3DMLUV-FIA solution. After discretizing rough surface with triangle patch, numbers of unknowns are 13066. The rough cutting layers $L_{s}=3$. Figure 5 shows the numerical result of the same rough surface when using 3DMLUV-FIA method and ACA method and the compression accuracy of the two method is $\varepsilon=0.001$. We can conclude from Figure 5 that the 3DMLUV-FIA method has the same effect as ACA method in $E$-plane and $H$-plane, which can prove the accuracy of 3DMLUV-FIA method.

Figure 6 shows the relationship between iterative steps and iterative deviation when applying three differences rough 
TABLE 1: Computation time and memory requirement.

\begin{tabular}{lccc}
\hline RMS height & $\begin{array}{c}\text { Memory (MB) } \\
\text { (ACA/3DMLUV) }\end{array}$ & $\begin{array}{c}\text { Compression time (s) } \\
\text { (ACA/3DMLUV) }\end{array}$ & $\begin{array}{r}\text { Computation time (s) } \\
\text { (ACA/3DMLUV-FIA) }\end{array}$ \\
\hline$h=0.04 \lambda$ & $186 / 169$ & $172.42 / 181.75$ & $28.35 / 10.19$ \\
$h=0.10 \lambda$ & $205 / 180$ & $213.25 / 218.23$ & $34.22 / 16.85$ \\
$h=0.15 \lambda$ & $233 / 188$ & $224.7 / 232.35$ & $41.26 / 30.56$ \\
\hline
\end{tabular}

surface and we can know from it that 3DMLUV-FIA can reach quick constriction even if the roughness of the rough surface changes (the permittivity of rough surface remains unchanged). And the iterative steps improve with the roughness enlargement.

Table 1 presents the difference of the memory requirements, the time of decomposing a matrix, and matrix solving time when using 3DMLUV method, ACA method, and 3DMLUV-FIA method. We can know from Table 1 that 3DMLUV has great advantage over ACA in memory requirements and computation time. From Figure 6 and Table 1, it can be included that 3DMLUV-FIA not only shows a high computation accuracy but also is highly efficient.

\subsection{Composite Scattering}

Case 3. Consider a hybrid 3DMLUV-FIA method for scattering from a $3 \mathrm{D}$ dielectric object above a $2 \mathrm{D}$ dielectric rough surface.

Given surface length $L_{x}=L_{y}=16 \lambda$, correlation length $l_{x}=l_{y}=\mathrm{cL}=0.5 \lambda$, RMS height $h=0.04 \lambda$, the relative permittivity $\varepsilon_{r 1}=2.5-j 0.18$, and $\varepsilon_{r 2}=5.4-j 0.04$, respectively. Let an average dielectric sphere with radius of $r=1.5 \lambda$ lie at altitudes of $d=2 \lambda$; the relative permittivity of sphere is $\varepsilon_{r, t}=2-j 3$.

After discretizing rough surface and object with triangle patch, numbers of unknowns are $N_{s}=56823, N_{o}=3388$. Facing such a big computation, the memory of traditional MOM is $5.4 \mathrm{G}$, while 3DMLUV is $685 \mathrm{M}$, which shows that 3 DMLUV has a great advantage in memory. Figure 5 presents the $H H$-polarized bistatic RCS only from dielectric rough surface. Figure 6 provides composite scattering from dielectric rough surface and dielectric sphere which lies above rough surface. We choose $E$-plane and $H$-plane for analysis. We produce 30 surfaces within different random surface roughness and calculate composite scattering with each rough surface and then take average result of the 30 statistics values.

Comparing Figure 7 with Figure 8, it is evident that the power of $H$-plane was enhanced (the RCS in $H$-plane increased, which leads to the power of $H$-plane enhanced) as the mutual interaction between rough surface and object. As for $E$-plane, whether object exists above the rough surface or not, from the direction of reflection, scattering peak appears in the figure because the degree of the rough surface is low. Iterative steps and iterative deviation are shown in Figure 8 applying 3DMLUV-FIA, which presents fast constrict speed.

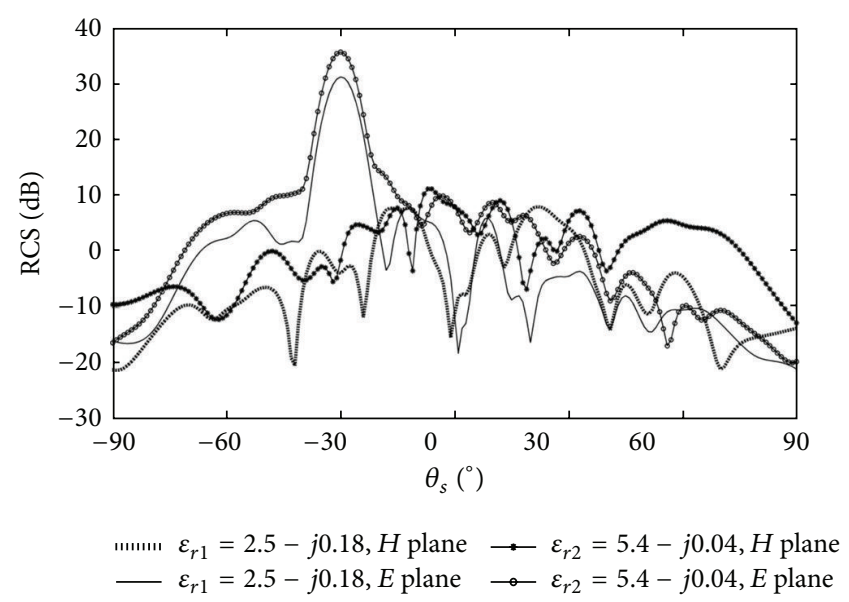

FIGURE 7: With different relative permittivity of rough surface $\varepsilon_{r 2}=$ $5.4-j 0.04, \varepsilon_{r 1}=2.5-j 0.18$, bistatic RCS from rough surface $(30$ average statistics values).

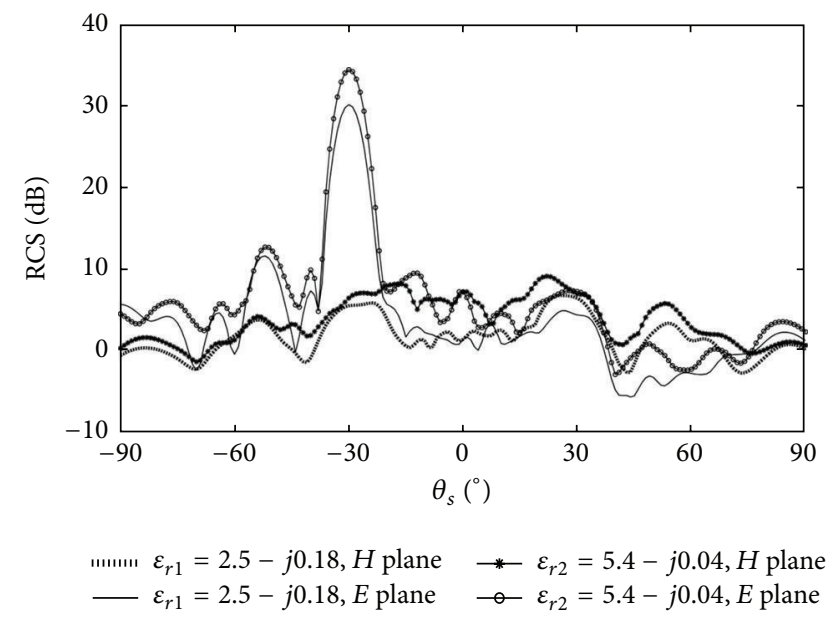

FIGURE 8: The relative permittivity of sphere is $\varepsilon_{r, t}=2-j 3$, the relative permittivity of rough surface is $\varepsilon_{r 1}=2.5-j 0.18, \varepsilon_{r 2}=5.4-$ j0.04, bistatic RCS from composite model of dielectric sphere and rough surface (30 average statistics values).

From Figures 8 and 9, we can know that 3DMLUV-FIA is highly efficient.

\section{Conclusion}

The 3DMLUV-FIA method is proposed to simulate the scattering from the dielectric objects. By investigating the bistatic 

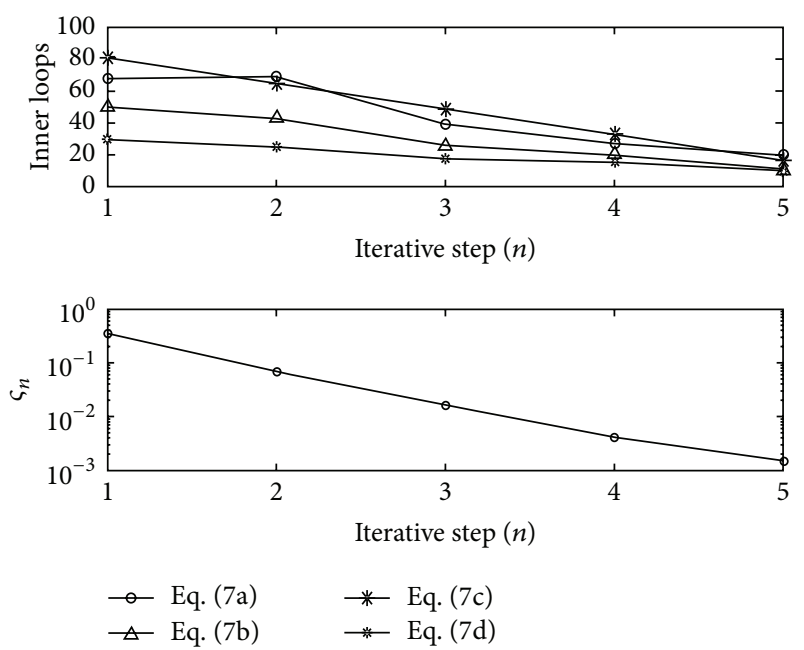

FIGURE 9: Inner loops and iterative deviation vary from iterative steps $n$.

electromagnetic scattering from the composite model of a 3D arbitrarily shaped object located above a $2 \mathrm{D}$ dielectric rough surface, this method is proved to be accurate and highly efficient.

To make the model more close to the actual real environment, more large and complex 3D object should be simulated. Considering the advantages of 3DMLUV method in memory and computation time and the characteristics easily parallelized and easily mixed with other methods, we can develop hybrid algorithm (such as hybrid 3DMLUVPO algorithm, 3DMLUV-ACA algorithm, 3DMLUV-FMM algorithm, etc.) for bigger size 3D composite scattering, using parallel multiprocessor computer and mass storage to solve large scale computational problems.

The research is limited to the combination of the target and rough surface scattering model, it will also be a very meaningful work to apply this method in scattering of buried target research.

\section{Competing Interests}

The authors declare that they have no competing interests.

\section{References}

[1] G. Q. Zhu, Q. F. Cao, and H. L. Yang, "Scattering from tablet above sinusoidal rough surface," Wuhan University Journal (JCR-Chinese Journal of Radioence Science Edition), 5 stage, 1996.

[2] P. Liu and Y.-Q. Jin, "Numerical simulation of bistatic scattering from a target at low altitude above rough sea surface under an EM-wave incidence at low grazing angle by using the finite element method," IEEE Transactions on Antennas and Propagation, vol. 52, no. 5, pp. 1205-1210, 2004.

[3] H.X. Ye and Y.-Q. Jin, "Fast iterative approach to difference scattering from the target above a rough surface," IEEE Transactions on Geoscience and Remote Sensing, vol. 44, no. 1, pp. 108-115, 2006.
[4] R. W. Xu, L. X. Guo, and Z. F. Wei, "Study on composite scattering from an arbitrary conducting object above PM sea surface with FEM/BIE," in Proceedings of the Cross Strait QuadRegional Radio Science and Wireless Technology Conference (CSQRWC '13), pp. 473-476, Chengdu, China, July 2013.

[5] J. Li, L. X. Guo, and Q. He, "Hybrid FE-BI-KA method in analysing scattering from dielectric object above sea surface," Electronics Letters, vol. 47, no. 20, pp. 1147-1148, 2011.

[6] A. W. Morgenthaler and C. M. Rappaport, "Scattering from lossy dielectric objects buried beneath randomly rough ground: validating the semi-analytic mode matching algorithm with 2D FDFD," IEEE Transactions on Geoscience and Remote Sensing, vol. 39, no. 11, pp. 2421-2428, 2001.

[7] M. El-Shenawee, C. Rappaport, E. L. Miller, and M. B. Silevitch, "Three-dimensional subsurface analysis of electromagnetic scattering from penetrable/PEC objects buried under rough surfaces: use of the steepest descent fast multipole method," IEEE Transactions on Geoscience and Remote Sensing, vol. 39, no. 6, pp. 1174-1182, 2001.

[8] J. Shin, A. W. Glisson, and A. A. Kishk, "Analysis of combined conducting and dielectric structures of arbitrary shapes using an E-PMCHW integral equation formulation," in Proceedings of the Antennas and Propagation Society International Symposium, vol. 4, pp. 2282-2285, Salt Lake City, Utah, USA, 2000.

[9] C. Yu, J. Yuan, and C. Gu, "Equivalent dipole-moment method for electromagnetic scattering by dielectric bodies," in Proceedings of the 3rd IEEE International Symposium on Microwave, Antenna, Propagation and EMC Technologies for Wireless Communications (MAPE '09), pp. 924-927, Beijing, China, October 2009.

[10] X. H. Yang, J. Hu, H. Y. Yao, and Z. P. Nie, “Solving scattering from 3D composite conducting and dielectric object by surface integral equation method," in Proceedings of the Asia-Pacific Microwave Conference (APMC '05), vol. 4, December 2005.

[11] B. M. Kolundzija, "Electromagnetic modeling of composite metallic and dielectric structures," IEEE Transactions on Microwave Theory and Techniques, vol. 47, no. 7, pp. 1021-1032, 1999.

[12] P. Ylä-Oijala and M. Taskinen, "Application of combined field integral equation for electromagnetic scattering by dielectric and composite objects," IEEE Transactions on Antennas and Propagation, vol. 53, no. 3, pp. 1168-1173, 2005.

[13] P. Yla-Oijala and M. Taskinen, "Improving conditioning of electromagnetic surface integral equations using normalized field quantities," IEEE Transactions on Antennas and Propagation, vol. 55, no. 1, pp. 178-185, 2007.

[14] R. F. Harrington, Field Computation by Moment Methods, Macmillan, New York, NY, USA, 1968.

[15] F.-S. Deng, S.-Y. He, H.-T. Chen, W.-D. Hu, W.-X. Yu, and G.-Q. $\mathrm{Zhu}$, "Numerical simulation of vector wave scattering from the target and rough surface composite model with 3-D multilevel UV method," IEEE Transactions on Antennas and Propagation, vol. 58, no. 5, pp. 1625-1634, 2010.

[16] P. Yla-Oijala and M. Taskinen, "Application of combined field integral equation for electromagnetic scattering by dielectric and composite objects," IEEE Transactions on Antennas and Propagation, vol. 53, no. 3, pp. 1168-1173, 2005.

[17] H. Ye and Y.-Q. Jin, "Parameterization of the tapered incident wave for numerical simulation of electromagnetic scattering from rough surface," IEEE Transactions on Antennas and Propagation, vol. 53, no. 3, pp. 1234-1237, 2005. 
[18] J.-J. Wu, "Simulation of rough surfaces with FFT," Tribology International, vol. 33, no. 1, pp. 47-58, 2000.

[19] L. Tsang, J. A. Kong, K. Ding, and C. O. Ao, Scattering of Electromagnetic Waves; Numerical Simulations, John Wiley \& Sons, New York, NY, USA, 2001. 


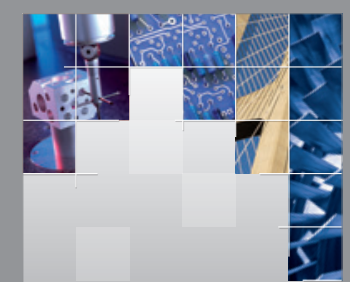

\section{Enfincering}
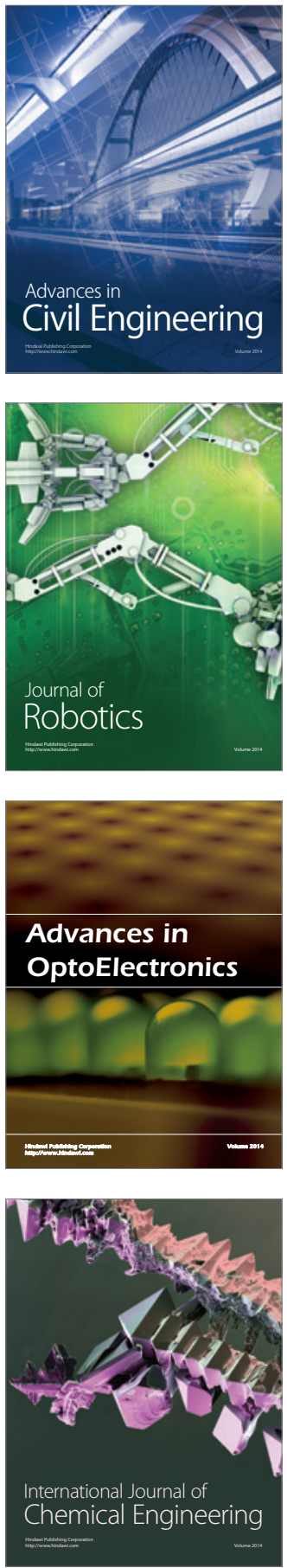

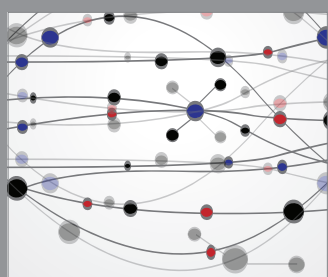

The Scientific World Journal

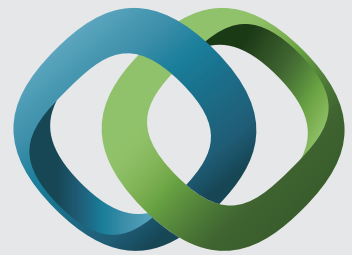

\section{Hindawi}

Submit your manuscripts at

http://www.hindawi.com
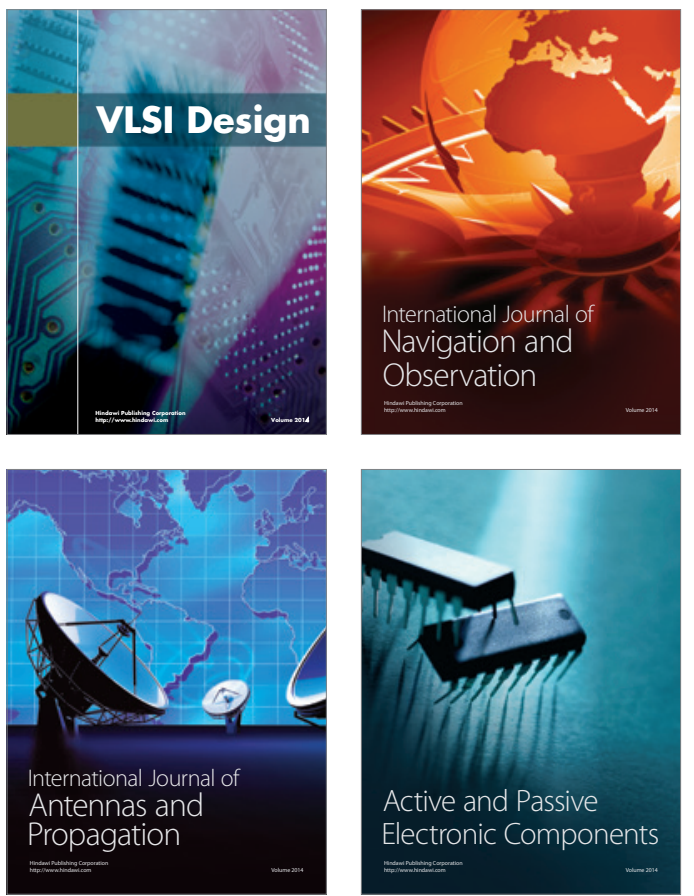
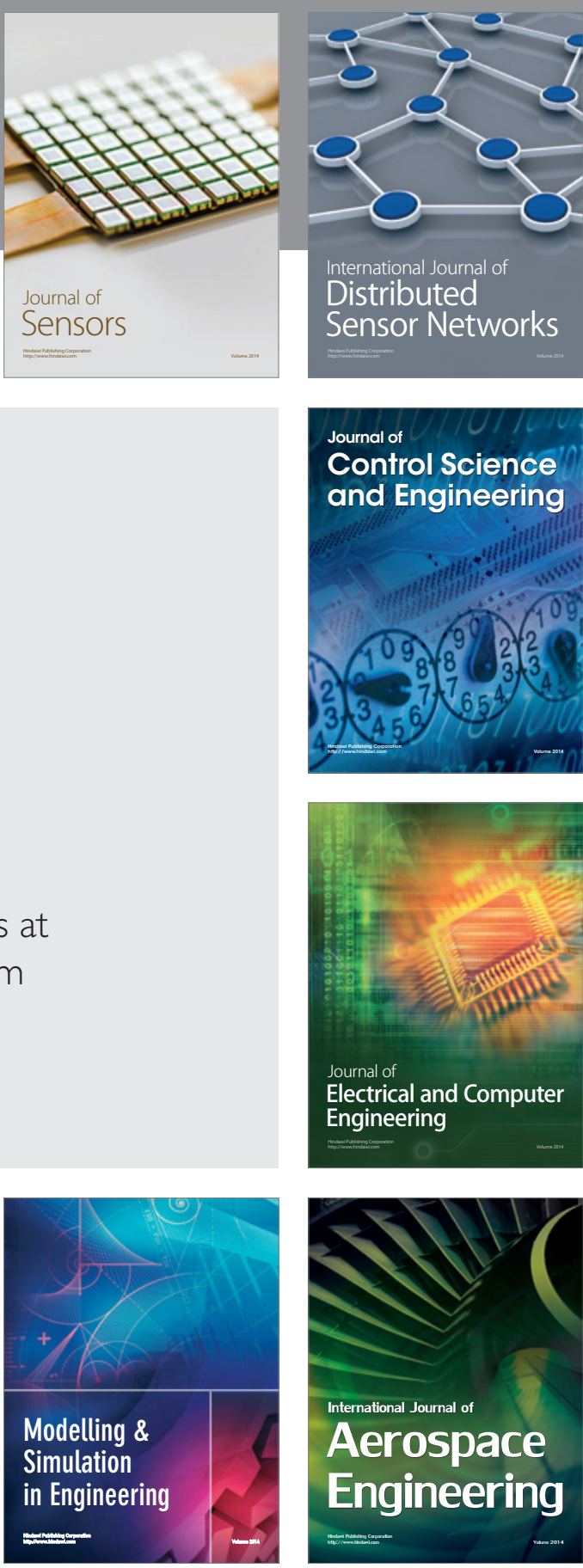

International Journal of

Distributed

Sensor Networks

Journal of

Control Science

and Engineering
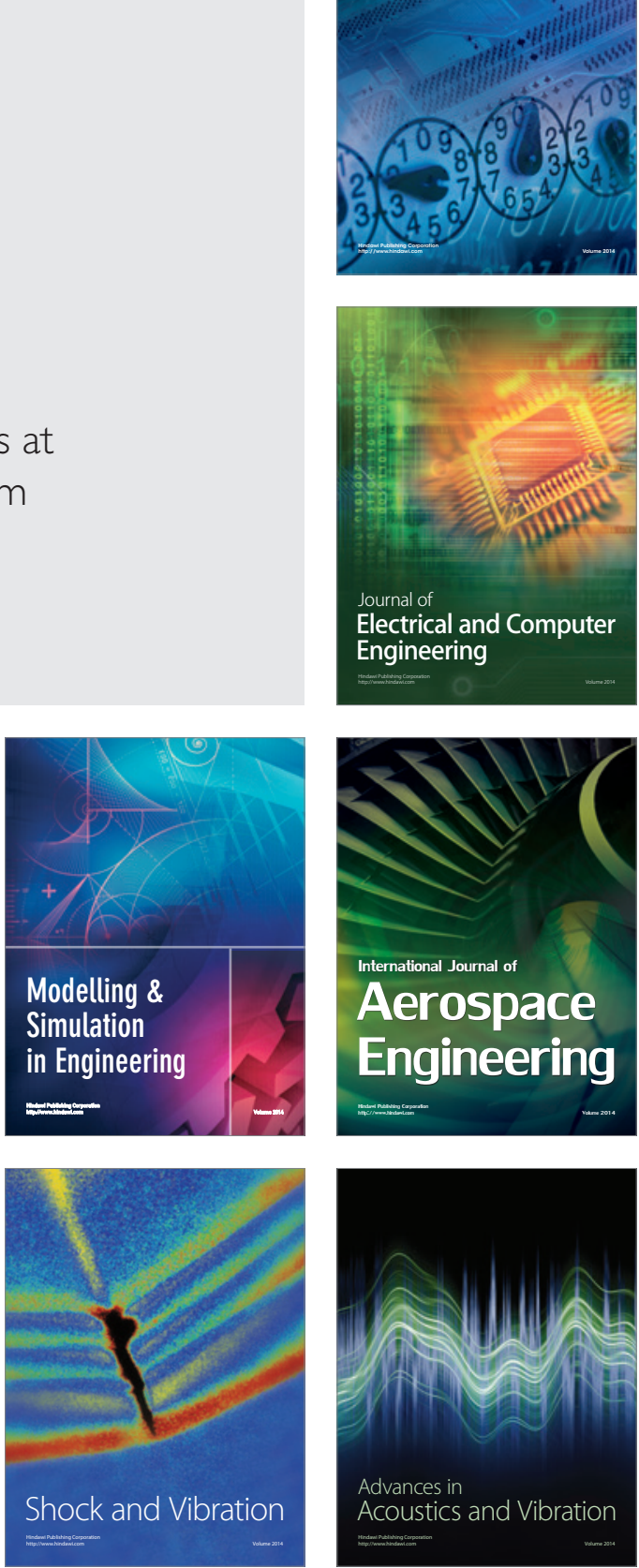